\title{
Los problemas de la normativa en el uso de la lengua catalana
}

\author{
JoAn Martí I Castell
}

UNIVERSIDAD ROVIRA I VIRGILI DE TARRAGONA

Una consecuencia de la anormalidad en la historia lingüística del catalán es la de la calidad en su uso, la de la competencia deficitaria de la propia comunidad catalanoparlante, que constituye una amenaza a la integridad del idioma.

La imposibilidad de usar habitualmente un idioma excluye obviamente la discusión sobre cómo se habla y cómo debe utilizarse. Por ello, el primer derecho que hay que exigir es el de su libre utilización. Pero difícilmente puede aspirarse a que sea suficientemente considerado desde el punto de vista social dentro y fuera de su área, si no se consigue un dominio satisfactorio en todos los niveles y registros, y en la práctica oral y escrita: dominio que se convierte precisamente en la señal de identidad más evidente que salva una lengua de su confusión híbrida con otra o de una relación de dependencia servil respecto a otra lengua dominante.

Es comprensible que, por lo que se refiere a la lengua catalana, habiendo sido explícitamente prohibida desde los poderes centralistas durante largos períodos, se sobrevalore la cuestión del uso, en perjuicio de su calidad: así sucedió en el siglo XIX y así ha sucedido y sucede en el siglo XX. Estratégicamente ha sido - y quizás es todavía - la vía correcta. Sin embargo, nos equivocaríamos si, en el contexto actual, en el que el catalán es lengua oficial, olvidásemos la vertiente de la recuperación de todos sus recursos por el interés exclusivamente de cuántos son los usuarios. El reconocimiento formal de la oficialidad del idioma borra la inquietud por su presencia real en la vida cotidiana y, en cambio, facilita que se ponga el énfasis en su calidad, especialmente porque en la educación escolar, en los grandes medios de comunicación social, en la administración, etc., se relaman orientaciones unitarias y 
útiles a los usuarios. La preocupación por el modo de usar una lengua subordinada es síntoma de que es necesaria en todos los niveles, de que se encuentra, pues en el camino hacia la efectiva normalidad. Pero existe el riesgo para su futuro de olvidar o no tener suficientemente en cuenta su carácter de subordinada.

Es obvio que, tanto respecto al primero como al segundo aspecto de la normalización linguiística -extensión del uso social y normativización-, hay opiniones enfrentadas y divergentes. Lo que es lógico, ya que ni en uno ni en el otro hay una meta objetiva que pueda presentarse como la línea de llegada ideal; nada que no sea discutible supone que la extensión del uso social de una lengua en contacto y con conflicto con otra con la que comparte la oficialidad es suficiente para poder hablar de normalidad. Ni nadie puede atribuirse que posee la llave para establecer definitivamente los modelos de lengua para las distintas funciones (hacemos expresamente abstracción de la autoridad democráticamente otorgada al Institut d'Estudis Catalans en ese ámbito).

Es un planteamiento erróneo pretender discernir qué es más conveniente: que una lengua sea hablada por muchos o por todos, aunque incorrectamente, o por pocos o por menos, pero correctamente. La normalización lingüística exige cantidad y calidad de uso simultáneamente. Hay que evitar el engaño de la ilusión por la extensión social y exigir que sea la comunidad entera quien use adecuadamente la lengua catalana.

$$
\text { 深深 }
$$

El catalán se mueve entre dos extremos en su uso que deben armonizarse mediante una aproximación necesaria: el culto y el literario, y el coloquial. Todas las lenguas presentan una distancia entre los diferentes registros, pero en los idiomas que no viven situaciones conflictivas es una distancia normal, útil, conveniente, para poder cumplir todas las estrategias, todas las funciones comunicativas. En el caso del catalán las divergencias suponen una anomalía especial, que va más allá de las deficiencias que presentan sus usuarios. En efecto, por el carácter que cada idioma tiene en cada comunidad de ser el elemento fundamental de identificación y de cohesión nacionales - a menudo, el elemento principal, como es el caso del catalán-, su consideración social se relaciona con la que se tiene de la nación. La corrección espontánea y mayoritariamente compartida en su uso se convierte en señal inequívoca de distinción. La historia de cualquier idioma llega a su punto culminante sólo cuando se ha establecido su codificación sólida, que funciona como referente aglutinador e identificador de la comunidad que se reconoce en él.

La Sección Filológica del Institut d’Estudis Catalans ha publicado (Barcelona 1991) unas declaraciones, La llengua i els mitjans de comunicació de 
massa. Declaració aprovada el 21 de setembre de 1990 (La lengua y los medios de comunicación de masa. Declaración aprobada el 21 de septiembre de 1990), cuyo primer punto dice:

«Todas las lenguas de cultura son identificadas por una modalidad común, que, a través de una cierta abstracción, permite a sus hablantes expresarse con espontaneidad y con comodidad, porque es la modalidad que se aprende en las escuelas, que se divulga a través de los medios de comunicación de masa,que se utiliza en los asuntos públicos y que cultivan los literatos y científicos. Esta es la modalidad legitimada de la lengua y todos pueden utilizarla e identificarse con ella, a pesar de que no contempla características locales que no pertenecen a la estructura esencial del sistema. Ello quiere decir, pues, que hasta cierto punto, en los niveles formales de la expresión y de la comunicación, todos renuncian a determinadas formas particulares de lenguaje, en beneficio de la unidad de la comunidad parlante. Esa modalidad es de todos, y al mismo tiempo no coincide completamente con el habla de cada uno de los que se sirven de ella como lengua propia. Esa modalidad es sancionada por la normativa gramatical y lexicográfica, que, a su vez, se basa en la historia literaria, la dialectología y el despliegue de la cultura." (Traducido del catalán).

洸深

La calidad de la competencia lingüística hay que entenderla básicamente como el grado de interferencias de idiomas distintos - particularmente de aquellos con los que puede haber conflicto-y, en otra dirección, como las connotaciones ideológicas que pesan en los hablantes: una lengua puede ser utilizada por toda una comunidad y, en cambio, ser considerada dependiente por naturaleza de otra(s). Por ello no es casual la insistencia vehemente en la necesidad de una rígida disciplina respecto a la normativa, que, a pesar de poder ser incómoda, ayuda decisivamente a neutralizar la condición de lengua subordinada que hoy el catalán todavía no ha perdido y que, en la perspectiva del marco político actual de los territorios donde es la única lengua propia, no perderá. La normativa del uso de la lengua catalana debe ser suficientemente sólida, segura y compartida como para que contribuya a la consolidación de la comunidad unitaria de los Países Catalanes.

Por otra parte, no podemos dejar de lado el riesgo de la dialectización de un idioma a causa del poco dominio social o de un uso no unitario o contravertido, dialectización que lo difumina a través de al menos tres vías: a) la de convertir la competencia en el nivel escrito-lector en un privilegio minoritario, b) la de empobrecerlo de recursos genuinos, aunque sea en nombre de la modernidad, y c) la de tratarlo como una lingua franca subyugada a una lengua dominante.

Las deficiencias en la expresión de una lengua en cualquiera de sus niveles son una amenaza que perpetúa una situación diglósica: tener que renunciar 
a recurrir a ella en beneficio de otra hace objetiva una distribución funcional, al margen de que el hablante crea o no en el condicionamiento del prestigio. Las dificultades en el uso de su idioma entre los catalanoparlantes se agravan en el ámbito de la producción escrita, precisamente la que de modo más decisivo colabora a la superación de las discriminaciones. Mientas la colectividad sufra el complejo de que es incapaz de recurrir con naturalidad a la lengua propia, será inevitable que tenga de ella un concepto especial, en el sentido de que no le resultará un instrumento que le sirve siempre y para todo, sino que de algún modo la hace sufrir con las dudas sobre la calidad de uso y con el temor de maltratarla.

$$
\text { 洸洸 }
$$

De la misma manera que no es normal que no aumenta el uso social del catalán, tampoco lo es el catalán que corrientemente se habla y el que (no) se escribe. El proceso de castellanización y la inexistencia de una larga tradición de usos formales en la época moderna justifican la realidad de la competencia linguística: con el considerable abandono de la lengua escrita, a los catalanes alfabetizados les han faltado durante muchos años las orientaciones que confirman continuamente la normativa y que constituyen su gramática viva. A los catalanes por alfabetizar, una escuela extraña a su lengua propia los ha sumergido en el desconocimiento de un punto de referencia común, más allá de las variaciones dialectales, y les ha imposibilitado un acceso normal a la cultura literaria en lengua catalana. Durante mucho tiempo hemos estado desprotegidos de la enseñanza formal y social que garantiza el uso espontáneo del idioma sin particulares obstáculos. Los resultados son fácilmente previsibles: pérdida de la unidad, pérdida de recursos, dialectalización creciente, anárquica diversificación del idioma; resultados que afectan a la genuinidad del catalán en todos sus niveles: fonético, ortográfico, morfológico, sintáctico y léxico. En buena parte de la comunidad catalana existe la conciencia de un déficit que provoca angustia en el acto de la comunicación. La inseguridad constante de si lo que se dice corresponde al sistema lingüístico o si, en cambio, es interferencia de otro genera una inquietud profunda al pensar que, sin quererlo, se puede traicionar, no ser leal al idioma minorizado. Por ello no es extraña la situación en que los interlocutores en el acto de comunicación se autocorrijan o corrijan a quienes les habla; incluso puede decirse que la comunidad catalana se distingue por un particular prurito de la corrección, con consecuencias como el temor de que nunca nada está completamente bien dicho o escrito, de que nunca nadie se expresa como convendría. Y con otra consecuencia con peligrosas connotaciones ideológicas no siempre advertida: la convicción de que el catalán es una lengua particularmente difícil de usar correctamente, de que es un idioma raro, complicado; esto justifica, entre otras razones, la tácita tolerancia entre los catalanoparlantes en lo que se refiere a su (in)competen- 
cia; tolerancia que convive con el deseo de poseer el dominio correcto del idioma.

Es indiscutible, en definitiva, que una lengua existe en la medida en que se usa; pero no lo es menos que la manera como se usa condiciona irremisiblemente su futuro.

\section{米米}

En la polémica entre los defensores de una lengua cercana a la que se habla coloquialmente y los que, en cambio, quieren una lengua libre de influencias extrañas -en Cataluña especialmente, claro está, influencias castellanas-, más próxima a la normativa gramatical, la historia lingüística del catalán (siglo XIX y primeros años del siglo XX) debiera habernos enseñado que ni hay que caer en un purismo intransigente no-realista, que se justifica sobre todo por la insistencia en que el idioma es la misma nación, idea que ha conducido a la sacralización de la lengua - lo que sin duda, hay que reconocerlo, ha colaborado decisivamente en su conservación, pero, al mismo tiempo, ha impedido e impide todavía tratarlo, manejarlo, adecuarlo de acuerdo con la realidad con naturalidad y sin complejos, superando las relaciones anormales que los catalanes tienen con su propia lengua-; ni hay que caer en un populismo simplista, demagógico y fácil. El punto 3 de la citada declaración del Institut d’Estudis Catalans dice:

«Justamente en el momento de iniciar una política de normalización lingüística -que obviamente tiene que confiar un papel importante a los responsables de los medios de comunicación de masa y a los educadores - han rebrotado, por razones extracientíficas, tendencias particularistas que parecían ya superadas. Asimismo ha surgido el afán de simplicación de la lengua que no hace sino acelerar el proceso de substitución, aparte del hecho de que se infringe la normativa en vigor.

Ambas corrientes - particularista y simplificadora- coinciden prácticamente, ya que ésta, en su reduccionismo, se apoya en soluciones locales que no se parecen a las de otras regiones y el hecho de fomentarlas provoca otro foco de secesión.» (Traducido del catalán).

Si en otras comunidades lingüísticas donde no hay situaciones de conflictos idiomáticos entre más de una lengua puede existir más fácilmente la inclinación por las tendencias innovadoras y más usadas socialmente que las conservadoras y más fieles a la preceptiva, la posición dialéctica en los Países Catalanes tiene que ser más equilibrada, porque se corre el riesgo de someter el sistema linguístico propio a los que tienen una situación privilegiada (español, francés e italiano). 
En realidad, el mismo hecho de que se produzca la polémica sobre los modelos de uso lingüistico en los términos es un síntoma evidente más de que somos una comunidad que vive anómalamente. De otro modo, el problema no preocuparía con la intensidad con que nos preocupa y, sobre todo, no tendría repercusiones fuera del ámbito estricto de los especialistas en temas lingüísticos.

En el proceso de aproximación armónica de la lengua literaria a la popular, hay que considerar que las interferencias del español son el resultado de la anormalidad histórica y no de una convivencia natural sin especiales hostilidades. Así pues, no se puede admitir indiscriminadamente todo lo que hoy utiliza la mayoría de catalanoparlantes: el criterio de la extensión social en el uso no puede ser el válido para la lengua catalana. En definitiva, hay que considerar su especificidad, en el sentido de que, habiendo vivido $-y$ viviendo todavía - tantos siglos con la intrusión prepotente de otras lenguas, se ha contaminado excesivamente de sus influencias, las cuales, por consiguiente, tienen que analizarse como un producto de procesos que han tendido a la eliminación del catalán. De lo que puede concluirse que es al menos imprudente abandonar precipitadamente soluciones genuinas en beneficio de las que las minorizan, con el argumento de que son poco o nada comunes entre la mayoría de los hablantes. No es buena solución el recurso expeditivo de permitir que el idioma, por un lado, no recupere todas sus posibilidades expresivas y, de otro, siga con los anormales préstamos del español, índice, al fin y al cabo, de la voluntad colonizadora de dialectalizar el catalán. Reproducimos, al respecto, el punto 5 y parte del 6 de la declaración del Institut d'Estudis Catalans:

«En efecto, la reducción de usos idiomáticos que algunos propugnan, con el pretexto de que la lengua sea más fácil, no hace sino empobrecerla. La exclusión alternativa de formas según criterios geográficos o regionales dificulta la comunicación entre catalanoparlantes y sugiere a los no catalanoparlantes una falta de unidad que no se corresponde con la realidad.

La lengua catalana ha conseguido un justo prestigio literario y cultural: ha dado figuras de primer orden en la historia de la literatura, es vehículo de una cultura destacada, es estudiada, conocida, traducida, valorada, estimada. Ahora le conviene consolidarse como instrumento eficaz de promoción y de integración social. Por eso cualquier medida que debilite su integridad es rechazable, no sólo porque pone en entredicho la unidad de la lengua. sino porque atenta, en general. contra el prestigio del catalán(...) Y ese atentado es grave porque el prestigio de la lengua condiciona su uso social e incluso su propia existencia (...) Recuperados determinados usos sociales de la lengua, sería suicida reducir sus capacidades expresivas. La necesaria adaptación funcional de la lengua a la nueva circunstancia 
no debe hacerse en perjuicio de su riqueza formal ni de su codificación actual. Enfatizar los problemas y las lagunas que muestra la normativa catalana (no mucho mayores que los de cualquier otra lengua), crear contramodelos gratuitos y presentados como productos salvadores, admitir y promover vulgarismos urbanos o localismos innecesarios, aceptar indiscriminadamente barbarismos a menudo de carácter argótico, confundir lo que puede ser el catalán básico con el catalán estándard o los distintos niveles lingüísticos, promover el espíritu polémico que todo ello motiva, no contribuye en absoluto a fortalecer la imagen pública de la lengua ni la cohesión de la comunidad.

Los factores mencionados pueden incluso afectar la nitidez de la lengua que se enseña en las escuelas, cuyo papel $\rightarrow$ no hay que olvidarlo- es, en el terreno lingüístico, primordial. Ahora se enseñan dos idiomas: uno en el que, al menos aparentemente, las cosas son claras, y otro en el que cada cual puede decir la suya y todo es problemático. Y esto no es admisible." (Traducido del catalán)

Además hay que tener un conocimiento técnico suficiente para proponer modelos de uso lingüístico, hay que entender que un idioma se utiliza en situaciones distintas que exigen registros igualmente diferentes: hay que ser conscientes de la significación real de la codificación linguística, más allá, como hemos visto, de la normativa que implica; no es lícito esgrimir el argumento simplista de que la preceptiva genuina es represiva (paradójicamente, no es extraño que quienes defienden el uso de las soluciones más próximas a los recursos coloquiales, lo hagan desde actitudes dogmáticas que pretenden privar a los usuarios de la libre elección: a menudo, pues, acaban siendo más inflexibles que la gramática que rechazan...).

Contra quienes puedan pensar que, además de forzado, es utópico no querer aceptar las soluciones más coloquiales no catalanas porque las alternativas del idioma no podrán imponerse, hay que decir que muchos recursos que parecían haberse arrinconado para siempre se han recuperado con relativa rapidez gracias a la escuela, a los medios de comunicación en general, pero, sobre todo, a la voluntad colectiva de hablar correctamente a la que hemos aludido, por el sentimiento de lealtad que provoca que el uso del catalán se convierta en un comportamiento más de respuesta nacionalista. Hoy ya prácticamente nadie dice adiós (adéu, adéu-siau), bocadillo (entrépà), casi (gairebé), ave (ve, ve baixa) busson (bústia), hasta (fins), etc., etc. Ya no suenan extrañas en el catalán oriental y central las vocales neutras finales de formas como frase, Balmes, cine, Vives, classe, Blanes, etc., etc., por citar una pequeñisima muestra de ejemplos de rectificaciones. Por lo tanto, hay que estar alerta de los peligros del catalán que se habla como modelo lingüístico para un idioma que ha vivido tantos siglos en la anormalidad y tan poco tiempo en la vía de la recuperación. 
Una de las funciones principales de la escuela es la alfabetización de la comunidad, en el sentido más amplio del término: tiene que enseñar la lengua en sus variantes diatópicas y geográficas y diastráticas o sociales, tiene que extender un estándard que la sociedad ha de consensuar. Pero tienen una función más incidente en este problema los medios de comunicación social: en primer lugar, porque se dirigen a la comunidad en su conjunto (a las personas de todas las edades, de ambos sexos, de todas las condiciones sociales, etc.); en segundo lugar, porque cubren todas las variantes de tipos de comunicación y de información y, por lo tanto, tienen en cuenta todos los registros. La enseñanza y los medios de comunicación social tienen, pues, especialmente, la responsabilidad de hacer competente a la población en el dominio del idioma, de presentarlo en sus variantes y de controlarlo permanentemente. No siempre ni todos los profesionales entienden la necesidad de resolver los déficits del idioma; no es extraño que consideren marginal a su responsabilidad entrar en esa polémica y no siempre son conscientes de que la disciplina en materia de uso lingüístico es imprescindible en cualquier sociedad, pero más todavía en aquellas cuyas lenguas propias se hallan expuestas a la fragmentación y a la dispersión. A pesar de todo, no faltan los reflejos de signo contrario: en algunos programas de radio o de televisión la corrección linguística es a menudo protagonista; algunos diarios o algunas revistas en catalán dedican sistemáticamente espacios a la reflexión sobre problemas de modelos de uso; últimamente se han publicado diversos libros de estilo destinados a orientar de manera uniforme a los profesionales de la prensa o a los correctores y asesores lingüísticos en general. Sin embargo, es oportuna aquí la recomendación que la declaración del Institut d'Estudis Catalans hace al respecto:

\footnotetext{
«Por ello, si bien hay que apoyar los trabajos de especialización funcional de la lengua, como los manuales o normas de estilo con los que distintas entidades públicas y privadas (editoriales, diarios y revistas, emisoras de radio y de televisión, entre otros) orientan a sus profesionales, hay que mirarlos con prevención cuando, en lugar de establecer nuevas convenciones concordantes con la normativa y relativas a cuestiones pendientes de resolución, proscriben elementos genuinos y normativos y fuerzan su substitución por otros que son nocivos a la salud de la lengua.» (Traducido del catalán)
}

\section{深深}

En los medios de comunicación social es donde se plasma de modo más vehemente la discusión sobre qué catalán hay que adoptar para difundirlo entre la comunidad. Y las opiniones son desiguales: hay quienes recriminan el uso de un catalán demasiado rígido y culto; la elección de las opciones menos corrientes y menos conocidas en la morfología, la sintaxis y el léxico; el recurso a un catalán muy próximo al que es normal en el nivel escrito literario, y, por ello, menos popular: se prefiere clowe (acahar, cerrar) frente a acabar. 
tancar; adresar-se (ir, dirigirse) frente a anar; proper (próximo) frente a pròxim o a la expresión que ve (que viene); mancar (faltar) frente a faltar; endegar (encaminar) frente a iniciar, signatura (firma) frente a firma; etc., etc,; una gran resistencia a la admisión de formas que han ganado terreno, algunas de las cuales incluso consideradas ya preceptivamente aceptables, como entregar, empleat (empleado), despedir. Junto a esas consideraciones, hay quienes culpan a los mass-media porque utilizan un catalán demasiado vulgar y coloquial, poco culto, innecesariamente concesivo.

Al margen de las opiniones sobre esa cuestión, se observa generalmente una fuerte inseguridad de algunos profesionales de la comunicación, a pesar de que ha habido una evolución positiva en la solución de los modelos, al menos en los principales medios de la prensa escrita y audiovisual. Por otra parte, la definición de un modelo de lengua es un trabajo delicado que ultrapasa su responsabilidad.

\section{棌棌}

El problema de la fijación del estándard del catalán, el más grave y urgente, no es estrictamente lingüístico, sino eminentemente sociolingüístico, ya que se trata de un registro - el más habitual, porque es neutro, en el sentido de supradialectal - que es como el puente entre la estricta normativa — que tiene que estar presente en el estándard-y los usos genuinos más extendidos; que se consolida mediante el uso total de los idiomas, siempre, por toda la comunidad y para todas las funciones: y el catalán no tiene ni mucho menos la presencia que permite establecerlo, reforzarlo y extenderlo:

"Mientras, tenemos que ser conscientes de que no hay más que una gramática y un diccionario, bastante flexibles ya, y que no es licito ni reducirlos ni mutilarlos." (Punto 8 de la declaración del Institut d'Estudis Catalans). (Traducido del catalán). 